\title{
Multiwavelength Search and Studies of Active Galaxies and Quasars
}

\section{A. M. Mickaelian}

NAS RA V. Ambartsumian Byurakan Astrophysical Observatory (BAO), Armenia

E-mail:aregmick@yahoo.com

\begin{abstract}
The Byurakan Astrophysical Observatory (BAO) has always been one of the centres for surveys and studies of active galaxies. Here we review our search and studies of active galaxies during last 30 years using various wavelength ranges, as well as some recent related works. These projects since late 1980s were focused on multiwavelength search and studies of AGN and Starbursts (SB). 1103 blue stellar objects (BSOs) on the basis of their UV-excess were selected using Markarian Survey (First Byurakan Survey, FBS) plates and Markarian's criteria used for the galaxies. Among many blue stars, QSOs and Seyfert galaxies were found by follow-up observations. 1577 IRAS point sources were optically identified using FBS lowdispersion spectra and many $A G N, S B$ and high-luminosity IR galaxies (LIRG/ULIRG) were discovered. 32 extremely high IR/opt flux ratio galaxies were studies with Spitzer. 2791 ROSAT FSC sources were optically identified using Hamburg Quasar Survey (HQS) lowdispersion spectra and many AGN were discovered by follow-up observations. Fine analysis of emission line spectra was carried out using spectral line decomposition software to establish true profiles and calculate physical parameters for the emitting regions, as well as to study the spectral variability of these objects. X-ray and radio selection criteria were used to find new AGN and variable objects for further studies. We have estimated AGN content of X-ray sources as $52.9 \%$. We have also combined IRAS PSC and FSC catalogs and compiled its extragalactic sample, which allowed us to estimate AGN content among IR sources as $23.7 \%$. Multiwavelength approach allowed revealing many new AGN and SB and obtaining a number of interesting relations using their observational characteristics and physical properties.
\end{abstract}

Keywords: active galactic nuclei - X-ray - infrared - radio - quasars - Seyfert galaxies - LINERs starburst galaxies - ULIRGs.

\section{Introduction}

The Byurakan Astrophysical Observatory (BAO) has always been one of the centres for surveys and studies of active galaxies, since mid-1950s, when V. A. Ambartsumian put forward his hypothesis on the activity of the galactic nuclei (Ambartsumian 1956a; 1956b; 1958; a detailed review on the development of Ambartsumian's ideas in this field is given by Harutyunian \& Mickaelian 2010). Very soon, B. E. Markarian 
started the first systematic survey for active galaxies (Markarian 1967; Markarian et al. 1989), using UV-excess method. Lists of candidate active galaxies were compiled also by M. A. Arakelian (1975) and M. A. Kazarian (Kazarian et al. 2010) using similar methods (Arakelian used the surface brightness criterion). Thousands of active galaxies were revealed and studied.

Since late 1980s, our research group was involved in search and studies of active galaxies using Markarian Survey observing material, then through optical identifications of IR and X-ray sources, statistical analysis of large multiwavelength catalogues and databases. We introduced multiwavelength (MW) research in BAO and accomplished a number of projects. Most of them were focused on MW search and studies of AGN and Starbursts (SB). The following directions may be mentioned:

- Search for QSOs and Seyferts among FBS Blue Stellar Objects (BSOs),

- Optical identification of IRAS PSC sources; active galaxies in the BIG sample,

- Optical identification of ROSAT FSC sources and BHRC AGN,

- Multiwavelength study of the bright AGN and Starbursts,

- Spectral and multiwavelength study of Markarian galaxies,

- IRAS PSC/FSC Combined Catalogue and its extragalactic sample,

- Study of extremely high IR/opt flux ratio galaxies with Spitzer,

- HRC/BHRC Combined Catalogue and its extragalactic sample,

- NVSS-FIRST cross-correlation: variable radio sources and their optical variability,

- ROSAT-NVSS AGN sample, search for new blazars,

- Multiwavelength study of blazars,

- Fine analysis of emission line spectra of active galaxies,

- DFBS and search for fainter Markarian and other active galaxies,

- AGN zoo and classifications by activity types,

- Activity of the galactic nuclei.

Many BAO collaborators have taken part in these projects and studies: $\mathrm{H}$. V.Abrahamian, K. S. Gigoyan, S. A. Hakopian, S. K. Balayan, L. K. Erastova, L. A.Sargsyan, P. K. Sinamyan, L. R. Hovhannisyan, H. V. Abrahamyan, G. M. Paronyan,G. S. Harutyunyan and G. A. Mikayelyan, as well as foreign colleagues: P. Véron,M.-P. Véron-Cetty, D. Engels, D. Weedman, J. Houck, E. Massaro, R. Nesci, C. Rossi, A. C. Gonçalves, D. Barry, H. Hagen, F.-J. Zickgraf, W. Voges, A. N. Burenkov, S.N. Dodonov, V. L. Afanasiev, A. V. Moiseev, R. Mujica, V. H. Chavushyan, D.-W.Xu, P. Prugniel, N. Gavrilovi'c, and others. 
Collaborations were established with Observatoire de Haute-Provence (OHP, France), Cornell University (Ithaca, N.Y., USA), Hamburger Sternwarte (HS, Germany), La Sapienza Università di Roma (Italy), Observatoire de Lyon (France), Max-PlanckInstitutfür Extraterrestrische Physik (MPE, Garching, Germany), Special Astrophysical Observatory (SAO, Russia), Instituto Nacional de Astrofisica, Optica y Electronica (INAOE, Mexico) and others.We have used many facilities, including ground-based and space telescopes / missions, databases, and dedicated software, such as BAO $1 \mathrm{~m}$ Schmidt and 2.6m, SAO (Russia) 6m, OHP 1.93m, Calar-Alto 2.2m, Palomar Observatory 5m, INAOE 2.12m, San Pedro Martir (Mexico) $2.12 \mathrm{~m}$ ground-based telescopes, IRAS, ROSAT and Spitzer space missions, DSS1/DSS2, SDSS, MAPS, Super COSMOS, DFBS, NED, Hyper LEDA, Vizier and other databases, Aladin, MIDAS, SMART and other software systems, Armenian Virtual Observatory (ArVO) and other VO tools. Dozens of papers have been published with the results of these studies and dozens of talks at important international meetings (IAU symposia and colloquia, JENAM / EWASS meetings, other dedicated meetings and workshops, etc.) and seminars have been given. Our group was awarded a number of research grants for accomplishment of these projects, including CRDF, ISTC, RA MES SCS, ANSEF, NASA, PICS / Jumelage, INSU ANR, DFG, DAAD grants and others.

Here we describe the most important projects related to search and studies of active Galaxies and their content among X-ray, IR and radio sources. Most important results, achievements and products are given at the end.

\section{Multiwavelength search and studies of active galaxies}

\subsection{Search for QSOs and Seyferts among FBS Blue Stellar Objects}

The $2^{\text {nd }}$ part of the First Byurakan Survey (FBS; Markarian et al. 1989) was conducted in 1987-1996 (Mickaelian 2000; 2008, and references therein) for selection and further study of blue stellar objects (BSOs) on the basis of the FBS observational material. The main purpose of this work was to discover new bright QSOs, Sy galaxies, other compact galaxies, as well as cataclysmic variables (CVs), white dwarfs (WDs), hot subdwarfs, HBB stars and other peculiar stellar objects. 1103 objects were selected(Figure 1), including 716 new BSOs. In total, 11 lists were published (Abrahamian \& Mickaelian 1996, and references therein) and the FBS BSOs catalogue is available at CDS in Strasbourg (Abrahamian et al. 1999). The completeness of the sample for objects with $B<16.5$ and $U-B<-0.5$ was estimated at about $67 \%$. Subsamples of candidate QSOs, WDs, CVs and other objects were constructed for their further detailed studies. Cross-correlation of FBS lists with ROSAT BSC and FSC (Voges et al. 1999; 2000) and radio catalogues NVSS and FIRST (Condon et al. 1998; 
Helfand et al. 2015) revealed candidate QSOs. Spectroscopic observations were carried out with BAO 2.6m, OHP $1.93 \mathrm{~m}$ and SAO $6 \mathrm{~m}$ telescopes, as well as SDSS data were used. We have discovered 12 new bright QSOs and S1 galaxies, including a bright $14^{m} \mathrm{NLS} 1$ at $z=0.118$. The total number of FBS QSOs is 42 (Mickaelian et al. 1999; 2001; Véron et al. 1999). We have constructed the most complete sample of bright QSOs $\left(B<16.6^{m}\right)$ over a large area $\left(2250 \mathrm{deg}^{2}\right.$, the subarea of FBS common with Palomar-Green (PG) survey with $|b|>30 \circ(G r e e n$ et al. 1986) and estimated their surface density as $0.012 \mathrm{deg}^{-2}$. The completeness of the bright quasar survey (BQS; Schmidt \& Green 1983) was revised to 53\%. New methods were suggested for accurate measurements of the positions, proper motions, magnitudes, colours and variability of FBS objects (Mickaelian 2004; Mickaelian \& Sinamyan 2010; Mickaelian et al. 2011).

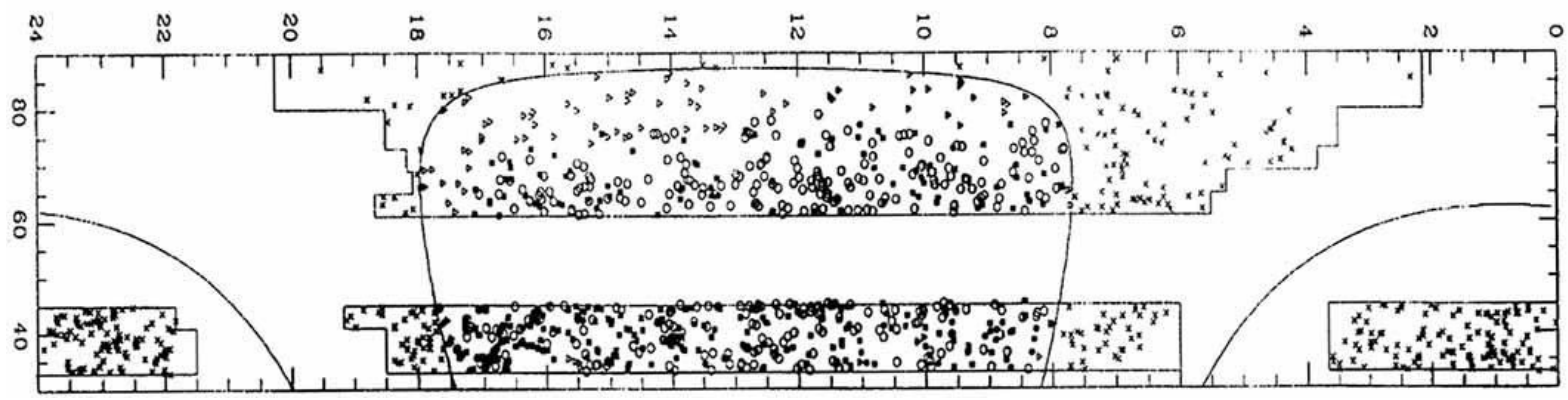

Figure 1. Distribution of 1103 FBS blue stellar objects on the sky. The lines show the Galactic plane $\left(b=0^{\circ}\right)$ and $b=+30^{\circ}$ latitude. Crosses -388 objects with $|b|<30^{\circ}$, triangles -97 objects with $|b|>30^{\circ}$ but outside the PG area, dots -276 objects detected by PG survey, circles -342 objects within the PG area but undetected.

\subsection{Optical identification of IRAS PSC sources and BIG sample}

A program of mass optical identifications of all IRAS (1988) PSC sources in a large $\left(1487 \mathrm{deg}^{2}\right.$ ) area on the basis of FBS low-dispersion spectra was conducted in the region with $+61 \circ<\delta<+90$ 。 at Galactic latitudes $|b|>15 \circ$ (Mickaelian 1995; 1997; Mickaelian \& Sargsyan 2004, and references therein).

Among the 1577 identified sources, there appear to be late-type stars, planetary nebulae, candidate QSOs, single and multiple galaxies and small groups. A new sample of IRAS galaxies was constructed, Byurakan-IRAS Galaxy (BIG) sample containing 1967 objects, including 789 previously known galaxies in this area and 1178 newly identified galaxies. Studies of BIG objects include spectroscopic followup for the brighter $(V<18)$ objects (redshift survey; Mickaelian et al. 1998; 2002; Balayan et al. 2001), discovery and study of new AGNs and ULIRGs, 2D spectroscopy of interacting and merging systems, search for obscured IRAS galaxies, and study of starburst (SB), AGN and interaction phenomena and their interrelationship. Spectral 
observations have been carried out using SAO (Russia) $6 \mathrm{~m}$, OHP $1.93 \mathrm{~m}$ and BAO 2.6m telescopes, as well as SDSS spectra are being used. 346 spectra for 229 BIG objects corresponding to 181 IRAS sources were obtained. Redshifts are in the range $0.008-0.173$ and FIR luminosity is $3 \times 10^{9}<\mathrm{L}$ fir $/ \mathrm{L}_{\odot}<7.5 \times 10^{12}$. Classification were made by diagnostic diagrams (Veilleux $\&$ Osterbrock 1987).
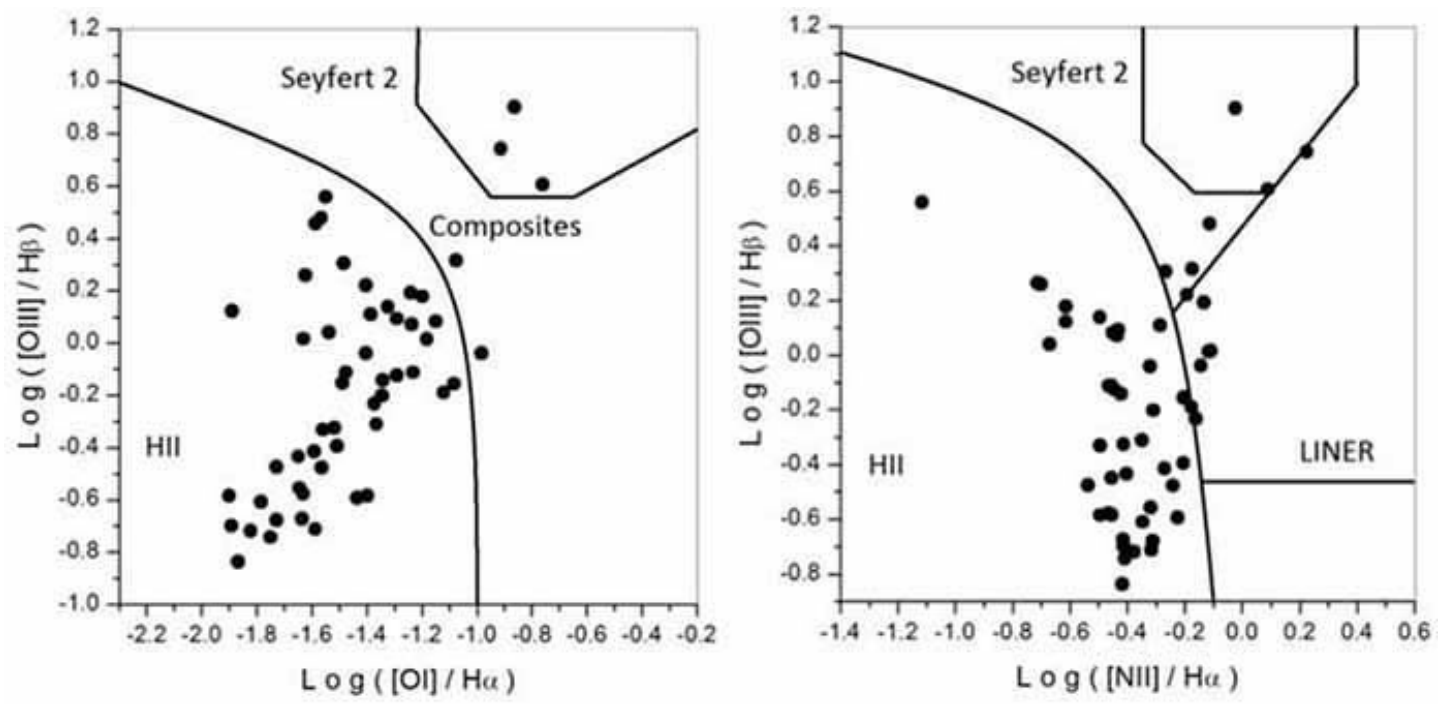

Figure 2. Diagnostic diagrams for BIG objects observed in SDSS. Many objects are located in the intermediate region between Seyferts, LINERs and HII galaxies showing composite spectrum.

BIG objects were grouped into several subsamples on the basis of their appearance, structure and nature: AGN (Sy galaxies, LINERS, composite-spectrum objects, etc.), high-luminosity IR galaxies, interacting/merging galaxies (Fig. 3, 4) and distant groups. The maximum IR luminosity for a single spiral galaxy was estimated (Mickaelian et al. 2001). High luminosity IR galaxies were revealed (Fig. 5).

\subsection{Optical identification of ROSAT FSC sources and BHRC AGN}

Two large works on optical identifications of ROSAT sources were carried out, using ROSAT BSC and FSC (Véron-Cetty et al. 2004; Mickaelian et al. 2006). A collaboration between Armenian (BAO), French (OHP), Hamburg quasar survey (HQS) and ROSAT teams with participation of scientists from INAOE (Mexico) and China was conducted to study the Hamburg-ROSAT identifications and to select NLS1 galaxies for further detailed investigation. Moreover, sources from the ROSAT FSC were identified and were observed as well. Beside NLS1 galaxies, X-ray sources identified with AGNs contain other interesting subsamples: QSOs, BLS1 galaxies, BLL, etc. An X-ray and optically flux-limited sample of $\sim 1000$ Seyfert 1 galaxies was created. 

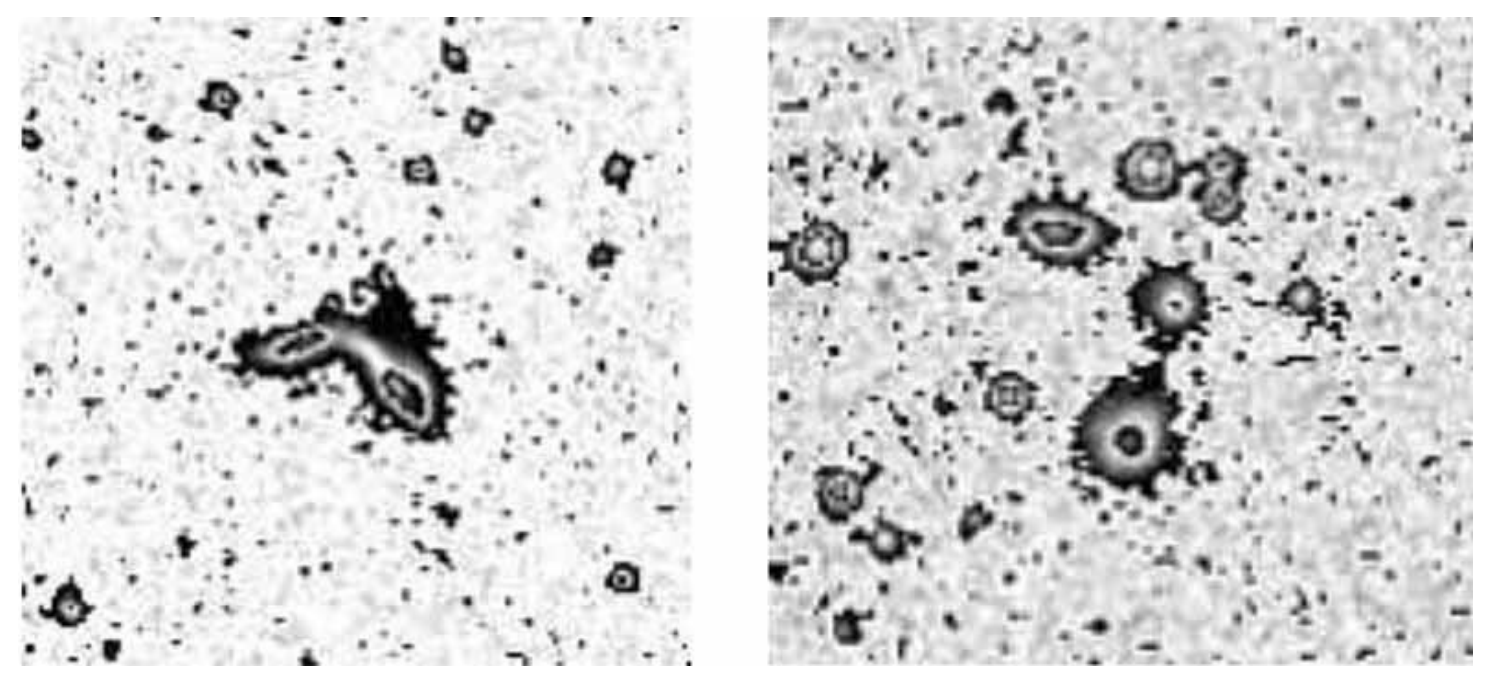

Figure 3. Two examples of multiple BIG objects IRAS 16358+6709 and IRAS 18380+8640. Image processing reveals signs of interactions between different formations. The physical groups have been confirmed by our spectroscopic observations; for the 2 components of IRAS $16358+6709$ system the average redshift and the dispersion are 0.053565 and 0.000165 , respectively, and for the 3 components of IRAS $18380+8640$ corresponding numbers are 0.077853 and 0.000041 .

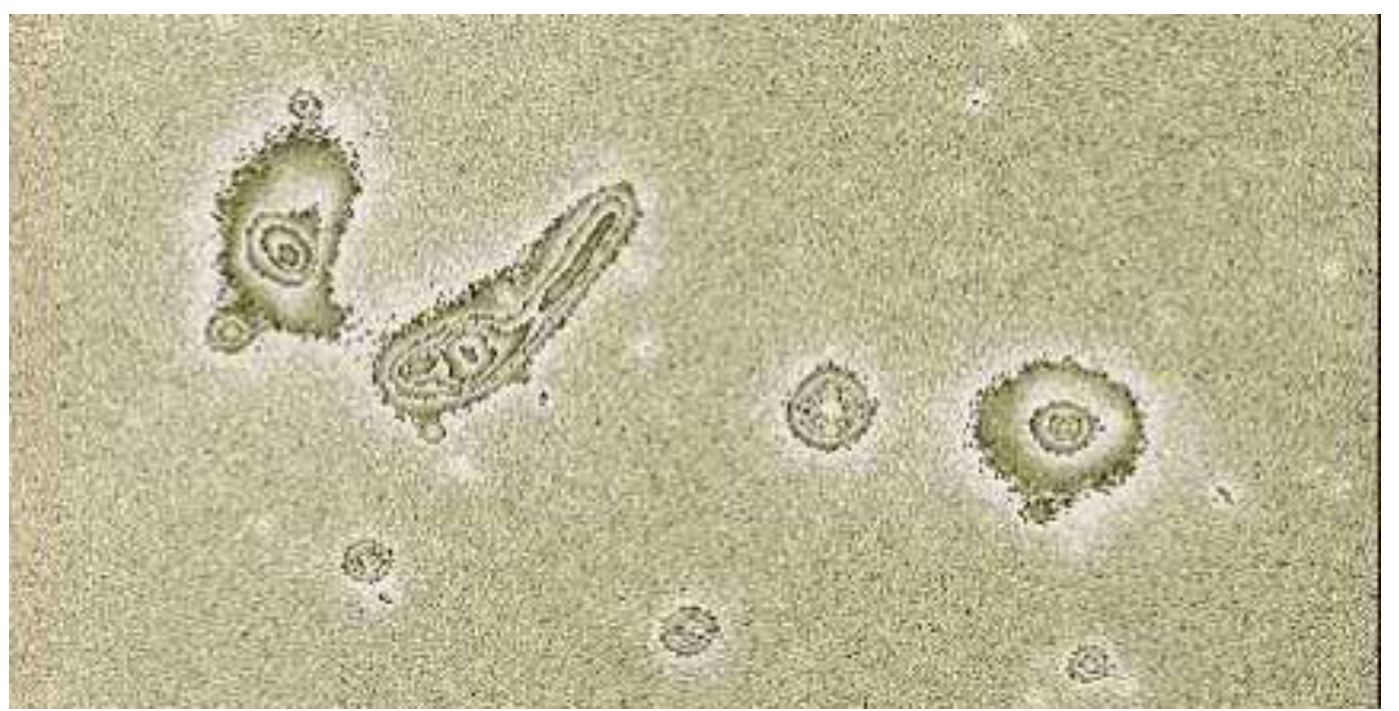

Figure 4. BAO 2.6m telescope ByuFOSC image of the interacting galaxy BIG284 = IRAS 12120+6838 obtained on 18.02.2002. The physical group have been confirmed by our spectroscopic observations. The 4 components have average redshift 0.060203 and the dispersion is 0.000702 .

\subsection{Multiwavelength study of bright AGN and Starbursts}

The bright AGN/SB sample from Véron-Cetty \& Véron (2010) Catalogue was retrieved for further statistical analysis. Some 10,000 objects are involved brighter than $17.5^{m}$. The project is aimed at accurate measurements of the magnitudes, including the core-host galaxy separation (as the Catalogue gives contaminated data), correction of absolute magnitudes (and hence, re-classification between QSOs and Seyferts), study of the morphology of AGN, and MW study of the whole sample aimed at revealing many relations by using various colour-colour and other diagrams. SEDs for bright AGN were built and studied (Mickaelian et al. 2012). 

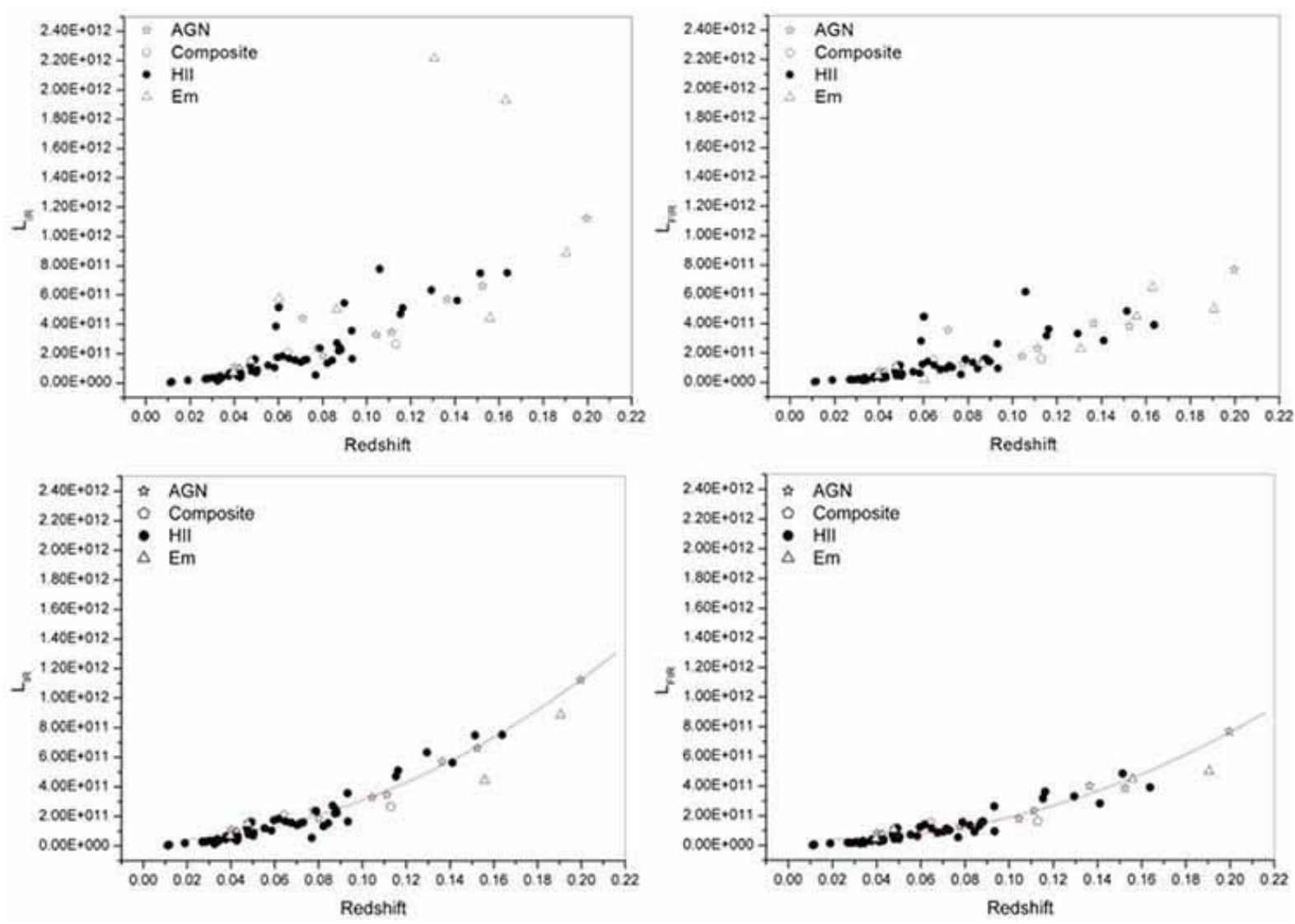

Figure 5. IR and FIR luminosities of BIG objects vs redshift. Top panels: most o fthe objects are located along a "main sequence", while there are 4 galaxies obviously having higher IR/FIR compared to what would be expected from the typical distribution. Bottom panels: Polynomial fits for the "main sequence" galaxies showing the accuracy of the correlation between IR/FIR and the redshift for this sample.

\subsection{Spectral and multiwavelength study of Markarian galaxies}

Markarian galaxies were published in a series of 15 papers and then in several catalogues (Mazzarella \& Balzano 1986; Markarian et al. 1989; Bicay et al. 1995; Petrosianet al. 2007). SDSS covered a significant area of Mrk survey. Markarian survey was digitized (Mickaelian et al. 2007; Massaro et al. 2008), and digital low-dispersion spectra are available for all Mrk galaxies. Using all available data on Markarian galaxies that appeared during the recent years, it is possible to carry out their homogeneous study to better characterize the sample in respect of various (including MW) parameters. We have classified 779 Mrk galaxies based on SDSS. These spectra give better classification and reveal fine details for these objects (Fig. 6). The distribution of galaxies by activity types is given in Table 1. We have built MW SEDs for all Mrk galaxies to study them using the whole electromagnetic range (Fig. 7). 



Figure 6. Examples of SDSS spectra of Mrk galaxies and their fine classification; Mrk 142 (Narrow Line Seyfert Galaxy, NLS1) and Mrk 1469 (S1.8 showing broad emission components both for $\mathrm{H} \alpha$ and H $\beta$ ).

Table 1. Distribution of 779 Mrk galaxies by activity types based on SDSS spectra

\begin{tabular}{|c|c|c|c|}
\hline \hline Activity type & Number & Activity type & Number \\
\hline HII & 533 & Sy1.2 & 21 \\
Composite (LINER/HII, Sy/HII, & 31 & NLS1 & 4 \\
Sy/LINER or Sy/HII/LINER) & & Sy1.0 & 4 \\
LINER & 12 & QSO & 2 \\
Sy2.0 & 4 & AGN (without an exact type) & 11 \\
Sy1.9 & 5 & Em (HII or AGN) & 52 \\
Sy1.8 & 8 & Abs & 65 \\
NLS1.5 & 5 & Star & 3 \\
Sy1.5 & 11 & Total & $\mathbf{7 7 9}$ \\
NLS1.2 & 8 & &
\end{tabular}

\subsection{IRAS PSC/FSC Combined Catalogue and its extragalactic sample}

We have compiled a joint catalogue of IRAS PSC and FSC by means of crosscorrelation of these two lists (Abrahamian et al. 2015). We have used a new tool for cross-correlations and as a result we obtained 73,770 associations. In addition, we have made cross-correlations with AKARI-IRC, AKARI-FIS and WISE catalogues. As a result, we created a catalogue with high positional accuracy and with 17 photometric measurements from 1.25 to $160 \mu \mathrm{m}$ range, providing a detailed catalogue for 345,163 IRAS point sources. 145,902 (42\%) of them are candidate galaxies, subject for our further studies of this large IR selected galaxy sample. The number of genuine galaxies (available from catalogs) is 78,240 and among them 18,567 are found to be AGN (from VCV-13, BZCAT v5 and SDSS QSOs catalogs), which makes their fraction 
23.7\%. Redshifts are available in total for 22,970 galaxies (mainly AGN and a small part of absorption galaxies).
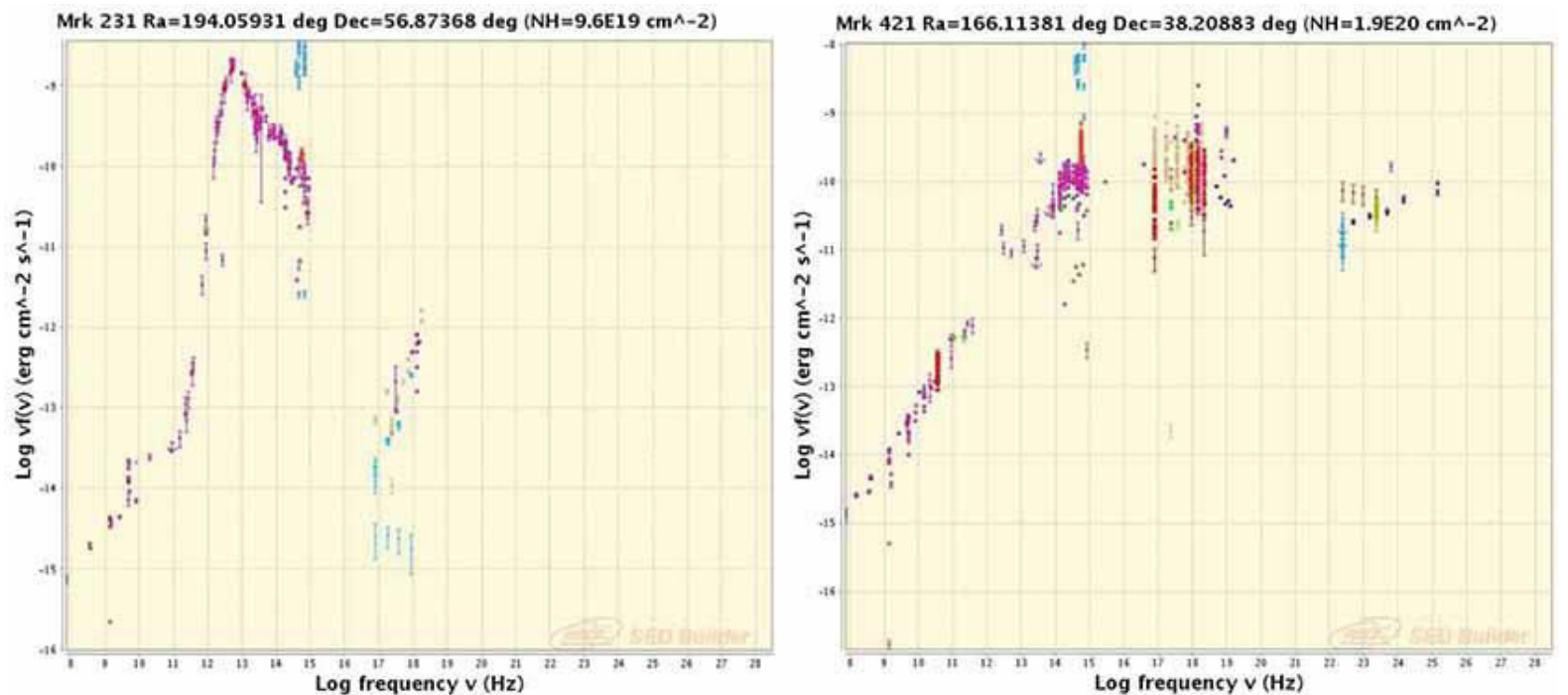

Figure 7. SEDs for two famous Markarian galaxies, Mrk 231 (the closest ULIRG,BAL QSO and most luminous IR galaxy in the Local Universe) and Mrk 421 (a blazar that is among the highest known energy sources), taken from ASI Science Data Centre, built by SED tool V2.0.

\subsection{Study of extremely high IR/opt flux ratio galaxies with Spitzer}

Based on the selection of IRAS FSC sources by means of cross-correlations with radio (FIRST) and optical (MAPS) catalogues, we have created a sample of extremely high IR/opt flux ratio galaxies and performed IR spectroscopy by Spitzer IRS (Sargsyan et al. 2008). Sources have $0.12<z<1.00$ and luminosities (erg.s ${ }^{-1}$ ) $43.3<\log [v \operatorname{Lv}(5.5$ $\mu \mathrm{m})]<46.7$, encompassing the range from local ULIRGs to the most luminous sources discovered by Spitzer at $z \sim 2$. Approximately half of the sources have luminosity dominated by an AGN and approximately half by a starburst (Fig. 8).

We analyzed the spectroscopic results for all galaxies observed with Spitzer IRS that also had total IR fluxes measured with IRAS, also using AKARI photometry. IR luminosities and SEDs from $8 \mu \mathrm{m}$ to $160 \mu \mathrm{m}$ were compared to PAH emission from SB galaxies or MIR dust continuum from $A G N$ at rest-frame wavelengths $\sim 8 \mu \mathrm{m}$ (Sargsyan et al. 2011). A sample of 230 galaxies was compiled based on their IRAS FSC fluxes to study their MW properties and carry out comparative analyses with other similar samples. We estimated the extinction for these 230 objects using SFRs calibrated from the PAH feature compared to UV flux, which showed that only $1 \%$ of the UV continuum typically escapes extinction by dust within a SB (Hovhannisyan et al. 2011). 

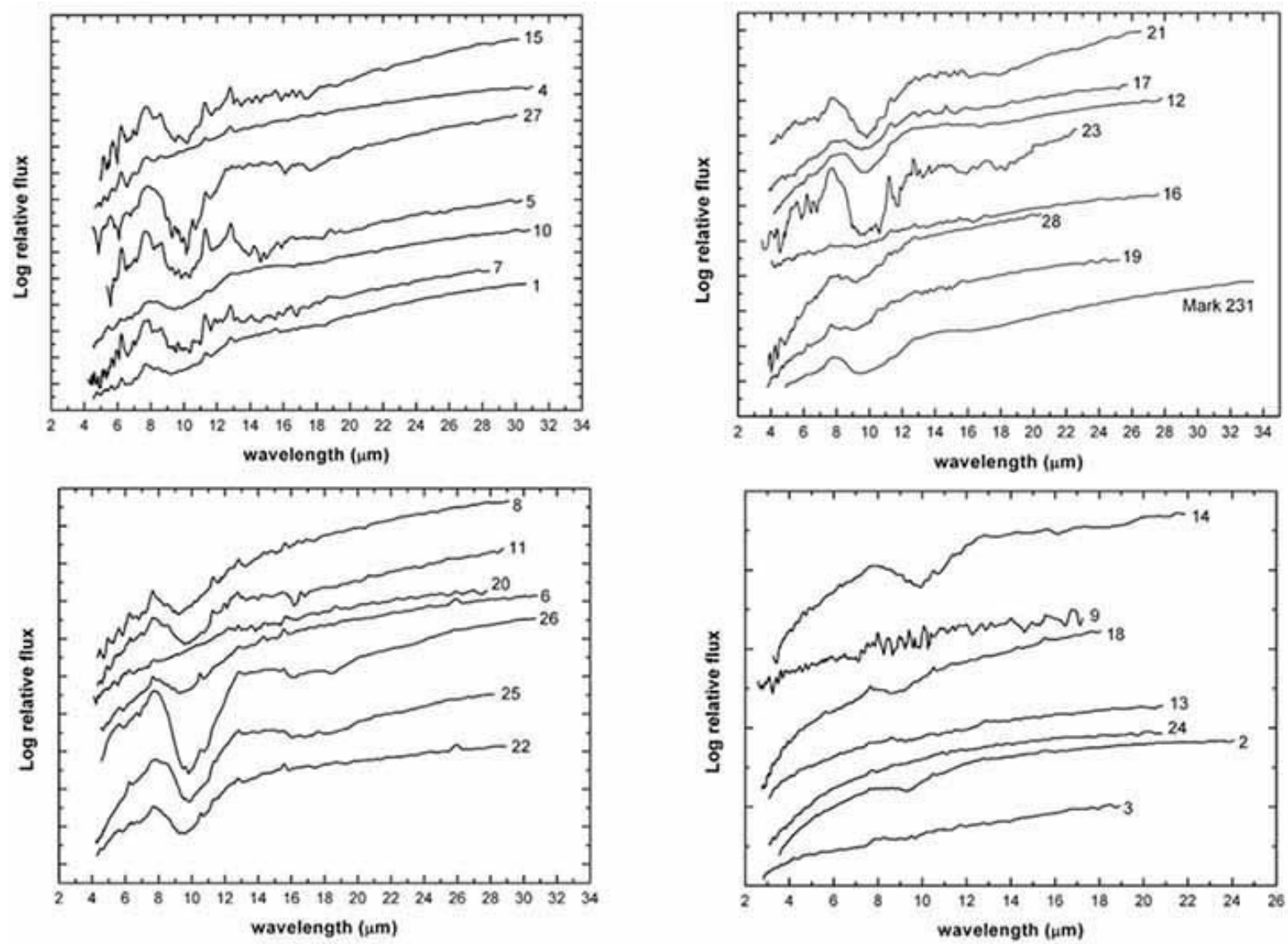

Figure 8. Spitzer IRS spectra of IRAS FSC sources in rest frame, ordered by $v L_{v}(5.5 \mu m)$. The lowest luminosity galaxy is given on the top. Sources of higher luminosities continue from top left to bottom right panels.

\subsection{HRC/BHRC Combined Catalogue and its extragalactic sample}

The joint catalogue of AGN selected from optical identifications of X-ray sources was created as a combination of two samples: Hamburg-ROSAT Catalogue (HRC; Zickgraf et al. 2003) and Byurakan-Hamburg-ROSAT Catalogue (BHRC; Mickaelian et al. 2006). Using the recent optical and MW catalogues we have revised both samples excluding false AGN and adding new genuine ones. Thus, a new large homogeneous complete sample of 4253 X-ray selected AGN was created. 3352 of them are listed in the Catalogue of QSOs and Active Galaxies (Véron-Cetty \& Véron 2010) and 387 also are in Roma BZCAT (Massaro et al. 2015). 901 candidate AGN are subject for further study. We classified 173 of these objects using their SDSS DR12 spectra(Fig. 9). Various activity types were revealed. A special emphasis is made on narrow-line Sy1.0-Sy1.5 galaxies and QSOs, as many of them have soft X-ray, strong Fell lines, and relatively narrow lines coming from BLR. We have retrieved MW data from recent catalogues and carried out statistical investigations for the whole AGN sample. An attempt to find connections between fluxes in different bands for 
different types of sources, and identify their characteristics thus confirming candidate AGNs have been carried out. We have analyzed X-ray properties of these sources to find a limit between normal galaxies and X-ray AGN (Paronyan et al. 2014a; 2014b; Mickaelian \& Paronyan 2014; Mickaelian et al. 2016; Paronyan \& Mickaelian 2016). Table 2 gives the grouped types of optical counterparts for $8037 \mathrm{X}$-ray sources.

Table 2. Types of optical counterparts for $8037 X$-ray sources and their fractions

\begin{tabular}{|c|c|c|}
\hline \hline Type & Number & $\mathbf{\%}$ \\
\hline Galaxy & 492 & 6.1 \\
AGN & 4253 & $\mathbf{5 2 . 9}$ \\
Star & 1800 & 22.4 \\
Unknown & 1492 & 18.6 \\
Total & $\mathbf{8 0 3 7}$ & $\mathbf{1 0 0 . 0 0}$ \\
\hline
\end{tabular}

After having more optical spectroscopic data from SDSS and combining AGN with Galaxy types (which may also contain AGN and SB), we have the following distribution by activity types (Table 3).

Table 3. Distribution of activity types for all HRC/BHRC extragalactic objects using SDSS DR12 spectra and other available data

\begin{tabular}{|c|c|c|}
\hline Activity type & Number & $\%$ \\
\hline BL Lac + HPQ & $320+13$ & 7.0 \\
QSO & 1309 & 27.6 \\
Sy1.0-1.5 & 1876 & 39.5 \\
Sy1.8-2.0 & 111 & 2.3 \\
Sy: & 37 & 0.8 \\
LINER & 21 & 0.4 \\
AGN & 82 & 1.7 \\
AGN: & 451 & 9.5 \\
Starburst / HII & 25 & 0.5 \\
Abs & 8 & 0.2 \\
Bright galaxy & 492 & 10.4 \\
Total & $\mathbf{4 7 4 5}$ & $\mathbf{1 0 0 . 0}$ \\
\hline
\end{tabular}




\subsection{NVSS-FIRST cross-correlation: variable radio sources}

NVSS (Condon et al. 1998) and FIRST (Helfand et al. 2015) have been carried out at the same wavelength $(21 \mathrm{~cm})$ or frequency $(1400 \mathrm{MHz})$, which gives a unique possibility to study radio variability of the common sources. The cross-correlation enabled us to find 556,282 radio sources present in both catalogs. Using the $3 \sigma$ criteria we distinguished 6,301 variable radio sources, and with certain limitations specified the 260 strongest radio variables. We cross-correlated these 260 sources with other catalogs at different wavelengths (APM, SDSS DR10, VCV-13, BZCAT, 2MASS, WISE). As a result, we obtained photometric data for optical, NIR, MIR and radio ranges for these 260 variable radio sources to study them in details (Abrahamyan \& Mickaelian 2014a; 2014b). The same will be done with all 6,301 sources, which we consider as AGN (1.1\% among associated radio sources).

\subsection{ROSAT-NVSS AGN sample}

$\mathrm{X}$-ray and radio radiation is typical of many active galaxies, but there are other $\mathrm{X}$-ray and radio sources as well. However, the cross-correlation of these two kind of sources results in mostly $A G N$, especially if we use not very deep surveys. We attempted to create an X-ray/radio AGN catalog and make its MW studies. We have cross-correlated ROSAT catalogs with NVSS one with a search radius of 30". 9,193 associations have been found. To distinguish AGN from the normal bright galaxies and clusters, Véron-Cetty \& Véron (2010) AGN catalog was used. 3,094 AGN were found. We were left with 6,099 X-ray/radio sources without an optical identification. Brighter objects are normal bright galaxies, while we believe that all faint ones are candidate AGN with some contamination of distant clusters. SDSS spectroscopic survey allows us classify objects by activity types, and a number of our candidate AGN is found to be present in SDSS. We attempt to find connections between the fluxes in different wavelength ranges, which will allow us to confirm AGN and blazars candidates and in some cases find new ones (Paronyan et al. 2014). If we consider all associated sources as AGN (given that both $\mathrm{X}$-ray and radio is present), then they make up $7.4 \%$ among 124,730 ROSAT $X$-ray sources and only $0.5 \%$ among $1,773,484$ NVSS radio ones. However, it is obvious that these numbers are underestimated, as we consider only one private method to reveal AGN.

\subsection{Multiwavelength study of blazars}

Blazars are the most interesting objects among AGN, both for their high luminosities and physical properties. We have conducted a project on MW studies of blazars based on the compilation of a homogeneous large sample of such objects ( 5000 blazars) and cross-correlations with available catalogues from $\gamma$-ray to radio. Roma- 
BZCAT (Massaro et al. 2015) and Véron-Cetty \& Véron (2010) Catalogue, as well as new SDSS QSOs are being used as a basis. Statistical analysis of the sample will be carried out to establish average properties of blazars and possibly find their subclasses. Optical and radio variability for the blazar sample will be established. A revised classification of blazars using the new data will be accomplished. MW SED for all blazars using automated software will be built. The evolution of various characteristics of blazars with luminosity and redshift will be studied. The luminosity evolution of various blazar sub-samples will be compared. Our data will make possible the study of their physical properties and establish the homogeneous definition of blazar phenomenon.
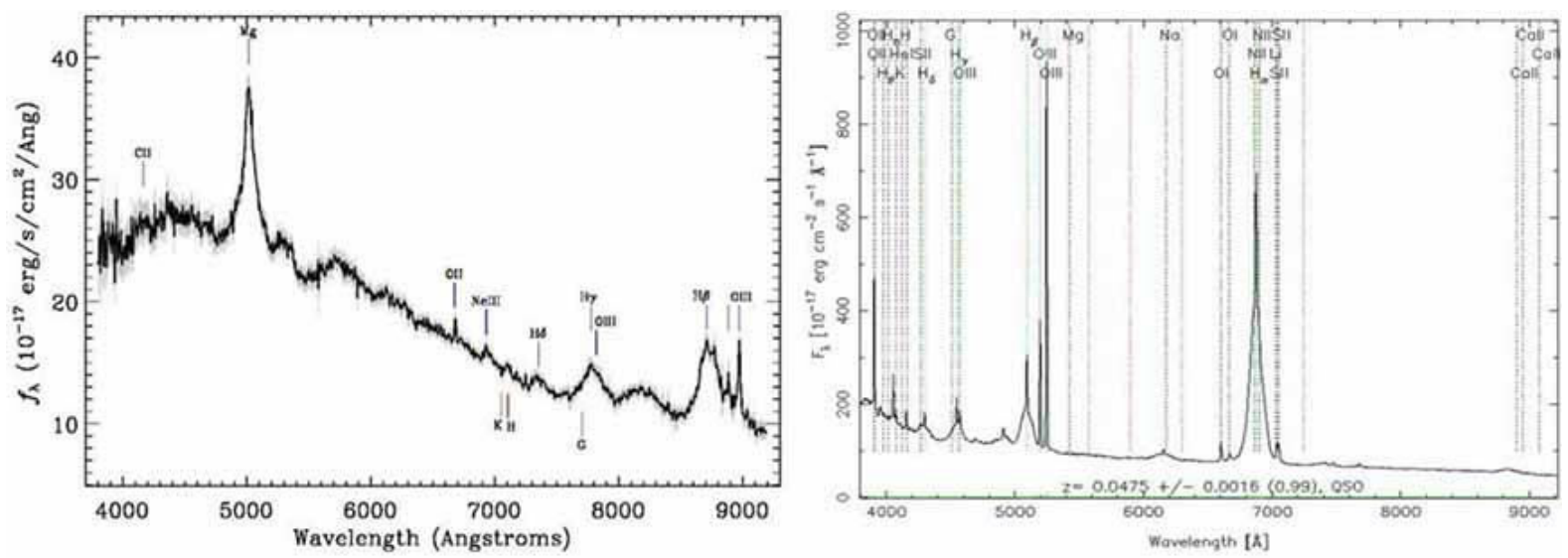

Figure 9. Example of SDSS spectra of HRC/BHRC AGN and their fine classification; left: a QSO also showing Fell lines on the left of $\mathrm{H}_{-}$typical of NLS1 and classified to NLQSO, right: S1.5 showing both narrow and broad emission lines with approximately equal intensity.

\subsection{Fine analysis of emission line spectra of active galaxies}

Most of type 1 AGN have contaminated spectral lines so that the broad component is overlapped on the narrow ones. A programme of fine analysis of emission line profiles and detailed spectroscopic classification of bright S1 galaxies (including NLSI ones) was conducted in collaboration with Véron \& Véron-Cetty (OHP, France). Itwas aimed at studying the differences between the classical BLS1 and NLS1 galaxies (Osterbrock \& Pogge 1985) and correlations between Fell and Balmer lines, Fe II and[OIII], and better understanding of the physics of AGN central regions. However, it appears that there is not a strict separation between the BLSI and NLSI galaxies (the line width limit at $2000 \mathrm{~km} \cdot \mathrm{s}^{-1}$ is arbitrary), and the intermediary objects may fill the gap. High-dispersion spectroscopy of $90 \mathrm{~S} 1 \mathrm{~s}$ having intermediate FWHMs was carried out at OHP $1.93 \mathrm{~m}$ telescope with $0.9 \AA$ /pix dispersion, the spectral resolution being3.2A. S1s from Véron-Cetty \& Véron (2010) with $V<16$ and $z<0.1$ were selected, including well-known objects (Fig. 10). 

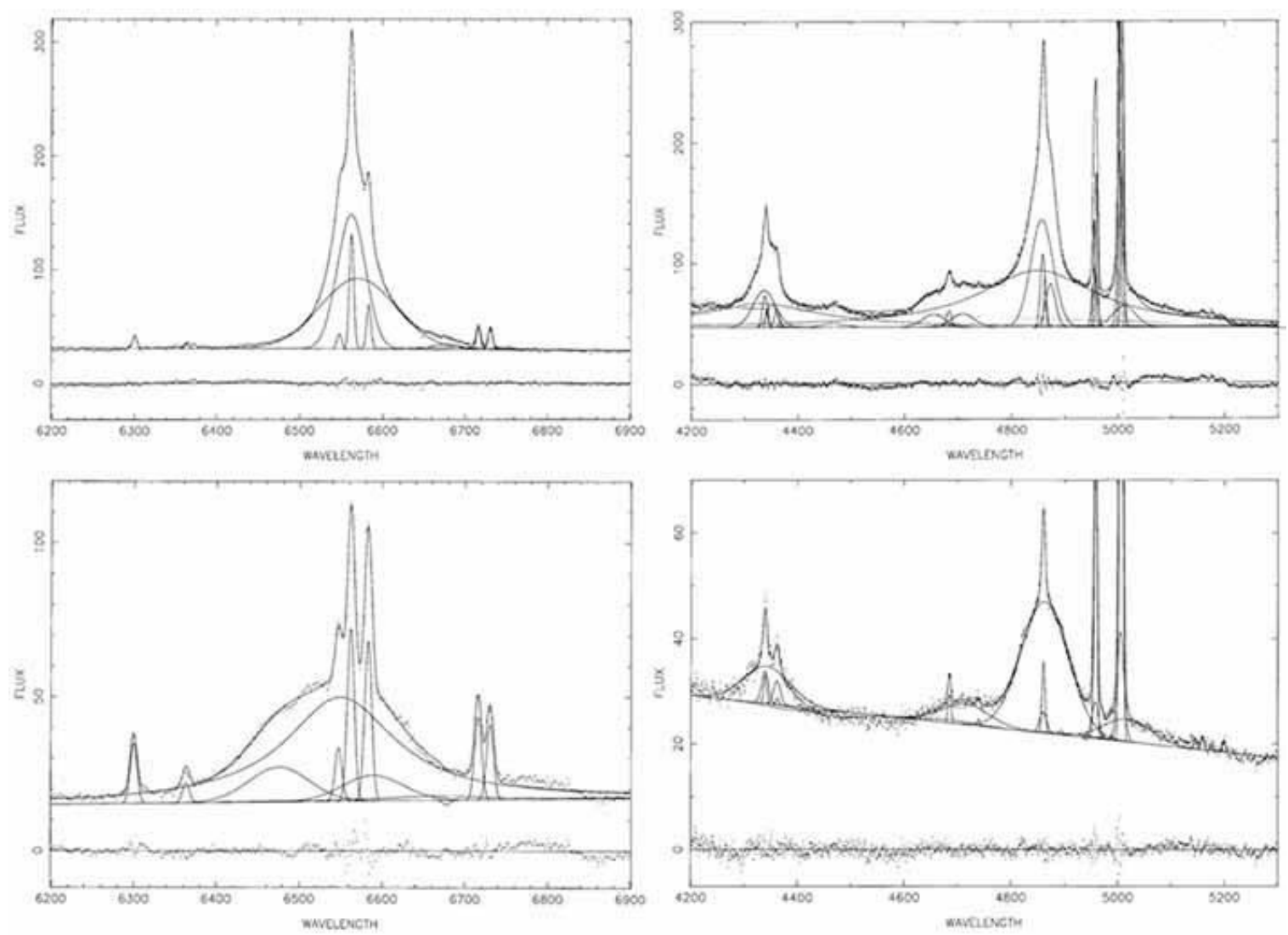

Figure 10. Fine analysis of the emission-line spectra of active galaxies with the dedicated software SPECTRAI. Examples of the results of the fitting in ranges around $\mathrm{H \alpha}$ (left panels) and $\mathrm{H} \beta$ (right panels). Top left panel: 3C 120 (an example of the easiest cases), top right panel: Mrk 509, bottom left panel: Mrk 926,bottom right panel: NGC 985.

The analysis was made using the special program SPECTRAI to fit the spectral lines profiles to the observed spectra (Véron et al. 1980). A comparison was made between the $\mathrm{H} \alpha$ and the $H \beta$ ranges observed in the framework of the same project. I n most cases, Balmer lines (and other permitted lines, such as Hel, Hell and Sill) are characterized by 2 broad components (2 Gaussian profiles or 1 Gaussian and 1Lorentzian) and 1 or 2 narrow components, and the forbidden lines ([NII], [OIII], [OI],[SII], etc.) by the same 1 or 2 narrow components. Fell and other Fe lines are often significant in the observed spectra. Together with the main emission lines (Balmer,[OIII] and [N II]), Hel, Hell, Ol, Sill and others are often present. There are objects with a number of high-ionization lines ([FeV]-[FeX], [AllI]-[AV], etc.) contrary to LINERS having strong low-ionization lines (Heckman 1980). We have constructed

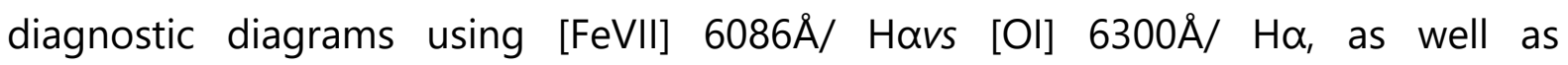

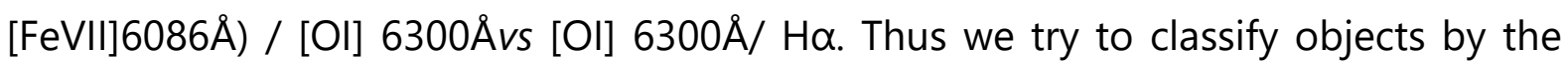
strength of their high-ionization lines. The distribution is well arranged and the objects are in as equence according to the ionization degree, with a good separation 
for those objects that we believe are of special interest. A study of variability of Kaz galaxies was also conducted (Kazarian \& Mickaelian 2007).

\subsection{DFBS and search for fainter Markarian and other active galaxies}

Even before the digitization project, some FBS spectra were scanned with the Machine Automatique de Mesures Astronomiques (MAMA, Observatoire de Paris, France) to reveal low-contrast spectral features. Five known QSOs were revealed, confirming the nature of these objects, including one discovered during our observations at OHP. Other objects have continuous spectra and may turn out to be QSOs, BL Lacs or DC WDs (Mickaelian et al. 2002).

Markarian Survey was accomplished with a goal of revealing new active galaxies and this was achieved by eye selection. We have digitized this survey plates and created the Digitized First Byurakan Survey (DFBS; Mickaelian et al. 2007; Massaro et al. 2008). Fig. 11 shows how efficient may be the low-dispersion spectra for optical identifications and search for new bright AGN.

The Armenian Virtual Observatory (ArVO; Mickaelian et al. 2009b) was created based on DFBS and many science projects have been discussed and tested (Mickaelian2007; Mickaelian et al. 2009a), including the search for new bright AGN and other active galaxies.
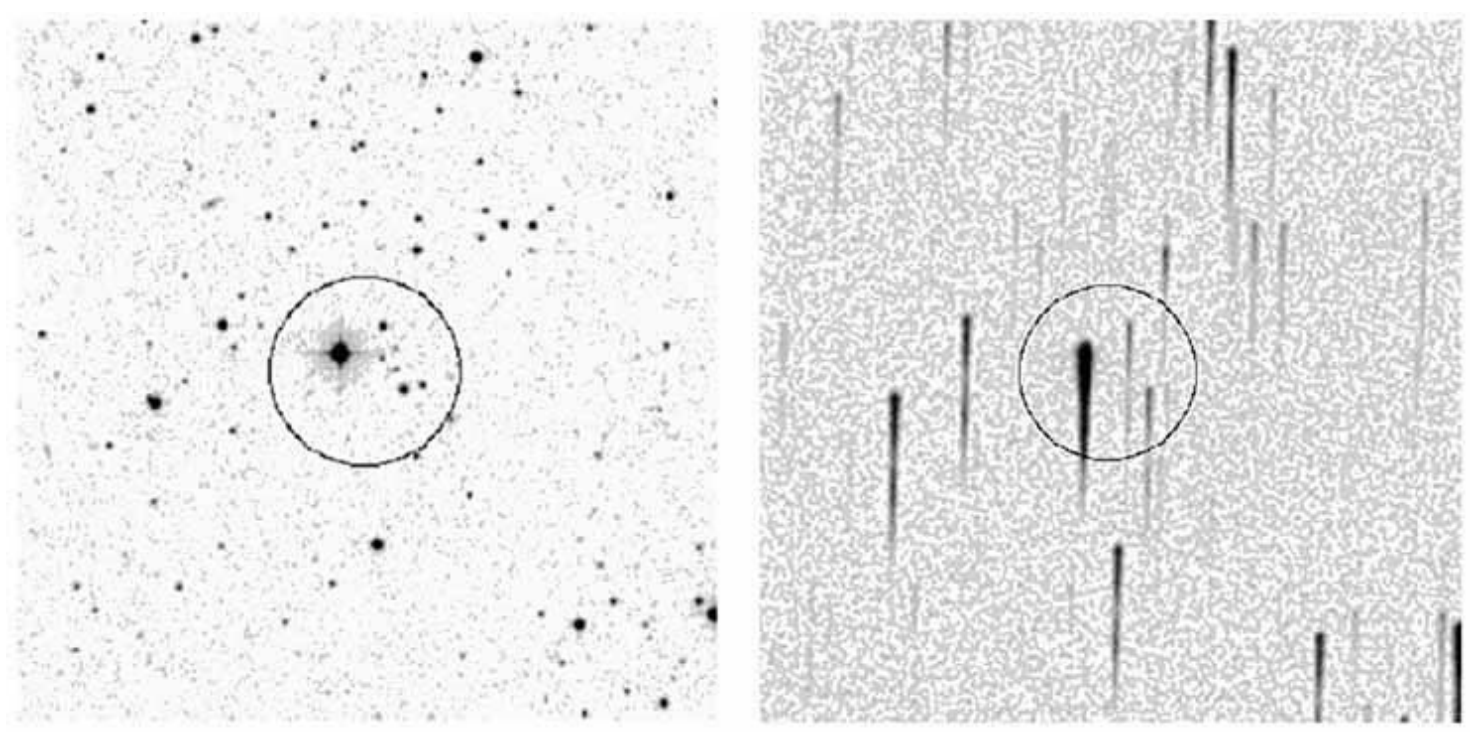

Figure 11. DFBS (low-dispersion spectra) significantly contributed in optical identifications of X-ray, IR and radio sources from ROSAT PSC/FSC, IRASPSC/FSC, NVSS, FIRST and other catalogues. Lowdispersion spectra reveal the genuine counterpart by SED and presence of emission and/or absorption lines, while direct images have no information rather than the positions and extension of objects. 


\subsection{AGN zoo and classification by activity types}

Many types of active galaxies have been introduced since 1950s. We have analysed all types and subtypes and suggested a joint scheme for Hyper LEDA database (Gavriloviceet al. 2007). Classifications from all available sources were taken into account. We introduced in Hyper LEDA information characterizing the nuclear and SB activity, as in particular the Véron \& Véron catalogue. We also described each type of AGN. It is now possible to retrieve an "activity type" for the Hyper LEDA galaxies having this attribute, and it is possible to select list of objects from constraints on "activity type". More detailed and justified version of such joint classification, as well as combination of MW data from -ray to radio is given in our recent publication (Mickaelian 2015).

\section{Summary and future projects}

A number of results were achieved by our search and studies of active galaxies at various wavelength ranges:

- discovery of 42 new bright AGN (QSOs and Seyferts) among the FBS BSOs, reestimation of the completeness of BQS (53\%) and estimation of the surface density of bright QSOs/AGN $\left(0.012 \mathrm{deg}^{-2}\right)$,

- optical identification of 1577 IRAS unidentified IR sources, including 1279 galaxies (BIG objects) and 287 stars (BIS), discovery of new bright AGN (Seyferts and LINERs) and ULIRGs among BIG objects, discovery of dozens of new pairs and multiple galaxies among IRAS sources, estimation of the maximum luminosity of a single spiral galaxy $\left(10^{12} \mathrm{~L}_{\odot}\right)$, study of interrelationship of AGN / interaction / starburst phenomena for IRAS galaxies,

- optical identification of 3212 ROSAT FSC X-ray sources; discovery of new bright AGN (QSOs and Seyferts) among X-ray sources, optical identification of 2791 ROSAT FSC X-ray sources (BHRC objects); estimation of the abundance of various types of objects among $\mathrm{X}$-ray sources, discovery of new types of $\mathrm{X}$ ray galaxies: interacting pairs and multiples,

- discovery of 32 highest IR/opt flux ratio extragalactic sources selected from IRAS FSC and observed with Spitzer, discovery of the highest IR/opt flux ratio (40-1000) extragalactic objects,

- a new sample of 330 IRAS / 2MASS / FIRST / SDSS selected galaxies, useful for comparative study of properties of IR sources,

- establishment of accurate magnitudes for AGN and the first statistical study of AGN catalogue, 
- discovery of the spectral variability of Akn 564 and Kaz 102; fine analysis of emission lines,

- homogeneous classification of 779 Markarian galaxies by activity types,

- based on the combined ROSAT BSC/FSC catalogue of optical identifications, we estimated AGN content of $X$-ray sources as $52.9 \%$,

- we have combined IRAS PSC and FSC catalogs and compiled its extragalactic sample, which allowed to estimate AGN content among IR sources as $23.7 \%$,

- definition of HINERs (High lonization Nuclear Emission-Line Regions),

- complete AGN classification for HyperLEDA database and elsewhere.

The results are published in the listed papers and books (DFBS, etc.), BAO, and ArVO web pages, the discovered objects are included in the most important databases (NED, Hyper LEDA, etc.), a number of catalogues are published in Vizier:

- First Byurakan Survey (FBS), 2nd Program (1999), Catalogue II/223

- FBS blue stellar objects DSS1/DSS2 astrometry (2004)

- Optically bright AGN in ROSAT-FSC (2004)

- Digitized First Byurakan Survey plate database (2005), Catalogue VI/116

- Optical identification of ROSAT-FSC sources (2006)

- Revised and updated First Byurakan Survey (FBS, 2008), Catalogue III/258

- IRS spectra of faint IRAS sources (2008)

- Proper motions of FBS blue stellar objects (2010)

- Variability of FBS blue stellar objects (2011)

- IR spectra and SEDs for starbursts and AGNs (2011)

- Study of 230 IRAS-FSC galaxies (2011)

- IRAS PSC/FSC Combined Catalogue (2015), Catalogue II/338.

Many new projects are possible based on our studies and using our data products (DFBS database, published catalogues, etc.). We give in Table 4 the summary of our projects on search and studies of active galaxies: short project name, years of accomplishment, objectives and number of involved objects. 
Table 4. Summary of projects on multiwavelength search and studies of active galaxies

\begin{tabular}{|l|c|l|r|}
\hline \multicolumn{1}{|c|}{ Project } & \multicolumn{1}{|c|}{ Years } & \multicolumn{1}{|c|}{ Objectives } & Objects \\
\hline FBS QSOs/Seyferts & $1986-2001$ & Bright QSOs and Seyferts & 1,103 \\
IRAS BIG sample & $1994-2010$ & AGN, SB, ULIRGs & 1,178 \\
ROSAT BSC/FSC sources & $2002-2006$ & Search for new AGN & 2,791 \\
Bright AGN & 2001 -pres. & Statistical studies & $\sim 10,000$ \\
Markarian galaxies & 2010 -pres. & Spectral and MW study & 1,515 \\
IRAS PSC/FSC & 2011 -pres. & Large IR galaxies sample & 145,902 \\
Spitzer ULIRGs & $2003-2010$ & High IR/opt flux ratio galaxies & 32 \\
HRC/BHRC AGN & 2010 -pres. & AGN content in X-ray & 4,253 \\
NVSS-FIRST & 2013 -pres. & Study of radio variability & 6,301 \\
ROSAT-NVSS & 2013 -pres. & Search for new AGN, statistics & 9,193 \\
MW study of blazars & 2014 -pres. & New blazars, definition & 3,561 \\
Fine analysis of spectra & $2001-2007$ & Physical properties of AGN & 90 \\
DFBS AGN & 2002 -pres. & New bright active galaxies & $\sim 10,000$ \\
Fine classification & 2006 -pres. & Accurate types and subtypes & $\sim 10,000$ \\
\hline
\end{tabular}

Acknowledgments. This work was supported by the RA MES State Committee of Science, in the frames of the research project No. 15T-1C257. This work was made possible in part by a research grant from the Armenian National Science and Education Fund (ANSEF) based in New York, USA (Grants astroex-4193 and astroex-4195). The authors thank to all colleagues for the fruitful collaboration and useful discussions.

\section{References}

Abrahamian, H. V.; Mickaelian, A. M. 1996, Ap 39, 315

Abrahamyan, H. V., Mickaelian, A. M.; Knyazyan, A. V., Harutyunyan, G. S. 2012, Proc. Conf.: 50 years of Cosmic Era: Real and Virtual Studies of the Sky. Yerevan, NAS RA, p. 223

Abrahamyan, H. V.; Mickaelian, A. M. 2014a, Proc. IAU Symp. 304: Multiwavelength AGN Surveys and Studies, Cambridge Univ. Press 304, 100

Abrahamyan, H. V.; Mickaelian, A. M. 2014b, Proc. IAU Symp. 304: Multiwavelength AGN Surveys and Studies, Cambridge Univ. Press 304, 102

Abrahamyan, H. V.; Mickaelian, A. M. 2016, Astronomical Surveys and Big Data, Eds. A. M. Mickaelian, A. Lawrence, T. Yu. Magakian. ASP Conf. Series, Vol. 505, p. 193 Abrahamyan, H. V.; Mickaelian, A. M. 2016, Proc. Armenian-Iranian Astronomical Workshop (AIAW), Eds.: A. M. Mickaelian, H. G. Khosroshahi, H. A. Harutyunian. Yerevan, NAS RA "Gitutyun" Publ. House, p. 208 
Abrahamyan, H. V.; Mickaelian, A. M.; Knyazyan, A. V. 2015, A\&C 10, 99; VizieR Online Data Catalog II/338

Abrahamyan, H. V.; Mickaelian, A. M.; Paronyan, G. M.; Mikayelyan, G. A.; Gyulzadyan, M. V. 2018, Astronomy \& Computing, 2018, in press.

Acero, F.; Ackermann, M.; Ajello, M.; et al. 2015, ApJS 218, 23

Alam, S.; Albareti, F. D.; Allende Prieto, C.; et al. 2015, ApJS 219, 12

Ambartsumian, V. A. 1956a, Proc. Fifth Conf. Problems of Cosmogony:

Radioastronomy, Acad. Sci. USSR, p. 413

Ambartsumian, V. A. 1956b, Izv. Acad. Sci. ArmSSR 9, 23

Ambartsumian, V. A. 1958, Proc. 11th Solvay Conf. on Physics: Structure of the Universe, ed. R. Stoops, Brussels, p. 241

Arakelian, M. A. 1975, Commun, BAO 47, 3

Balayan, S. K.; Hakopian, S. A.; Mickaelian, A. M.; Burenkov, A. N. 2001, Astron. Letters 27,284

Bianchi, L.; Herald, J.; Efremova, B.; et al. 2011, Ap\&SS 335, 161

Bird, A. J.; Bazzano, A.; Bassani, L.; et al. 2010, ApJS 186, 1

Cabanela, J. E., Humphreys, R. M., Aldering, G., et al.: 2003 PASP 115, 837

Condon, J. J., Cotton, W. D., Greisen, E. W.; et al.: 1998 AJ 115, 1693

Cutri, R. M.; Skrutskie, M. F.; van Dyk, S.; et al. 2003, IPAC/California Institute of Technology

Cutri, R. M.; Wright, E. L.; Conrow, T.; et al. 2013, AlIWISE Data Release, IPAC/Caltech, VizieR Catalog II/328

D'Elia, V.; Perri, M.; Puccetti, S.; et al. 2013, A\&A 551, A.142

Erastova, L. K.; Mickaelian, A. M. 2016, Astronomical Surveys and Big Data, Eds. A. M. Mickaelian, A. Lawrence, T. Yu. Magakian. ASP Conf. Series, Vol. 505, p. 242

Erastova, L. K.; Mickaelian, A. M. 2017, Astron. Astrophys, Transaction, Vol. 30, 2017, in press.

Gavrilovi'c, N.; Mickaelian, A. M.; Petit, C.; Popovi'c, L. 'C.; Prugniel, P. 2007, Proc. IAU Symp. 238: Black Holes from Stars to Galaxies - Across the Range of Masses. Cambridge Univ. Press, p. 371

Green, R. F.; Schmidt, M.; Liebert, J. 1986, ApJS 61, 30

Gregory, P. C., Scott, W. K., Douglas, K., Condon, J. J. 1996, ApJS 103, 427

Gyulzadyan, M. V.; Mickaelian, A. M.; Abrahamyan, H. V.; Paronyan, G. M. 2016, Astronomical Surveys and Big Data, Eds. A. M. Mickaelian, A. Lawrence, T. Yu. Magakian. ASP Conf. Series, Vol. 505, p. 162

Gyulzadyan, M. V.; Mickaelian, A. M.; Abrahamyan, H. V.; Paronyan, G. M. 2016, Proc. Armenian-Iranian Astronomical Workshop (AIAW), Eds.: A. M. Mickaelian, H. G. Khosroshahi, H. A. Harutyunian. Yerevan, NAS RA "Gitutyun" Publ. House, p. 227 
Gyulzadyan, M. V.; Mickaelian, A. M.; Abrahamyan, H. V.; Paronyan, G. M.; Mikayelyan, G. A. 2017, Proc. International Conference "Non-Stable Universe: Energetic Resources, Activity Phenomena and Evolutionary Processes", Eds. A. M. Mickaelian, H. A. Harutyunian, E. H. Nikoghosyan, ASP Conf. Series, Vol. 511, p. 192 Hagen, H.-J.; Engels, D.; Reimers, D. 1999, A\&AS 134, 483 Hambly, N. C.; MacGillivray, H. T.; Read, M. A.; et al. 2001, MNRAS 326, 1279 Harutyunian, H. A.; Mickaelian, A. M. 2010, Proc. Conf.: Evolution of Cosmic Objects through their Physical Activity. Yerevan, NAS RA "Gitutyun" Publ. House, p. 134 Harutyunyan, G. S.; Mickaelian, A. M. 2012, Proc. Conf.: 50 years of Cosmic Era: Real and Virtual Studies of the Sky. Yerevan, NAS RA, p. 157

Harutyunyan, G. S.; Mickaelian, A. M. 2014a, Proc. IAU Symp. 304: Multiwavelength AGN Surveys and Studies, Cambridge Univ. Press 304, 68

Harutyunyan, G. S.; Mickaelian, A. M. 2014b, Proc. IAU Symp. 304: Multiwavelength AGN Surveys and Studies, Cambridge Univ. Press 304, 383

Helfand, D. J.; White, R. L.; Becker, R. H. 2015, ApJ 801, 26

Hovhannisyan, A.; Sargsyan, L. A.; Mickaelian, A. M.; Weedman, D. W. 2011, Ap 54, 147

IRAS, 1988, Joint IRAS Scienve Working Group. IRAS PSC, Version 2.0, NASA RP-1190 Ishihara, D.; Onaka, T.; Kataza, H.; et al. 2010, A\&A 514, 1

Kazarian, M. A.; Adibekyan, V. Zh.; McLean, B.; Allen, R. J.; Petrosian, A. R. 2010, Ap 53, 57; Vizier On-line Data Catalogue VII/254

Kazarian, M. A.; Mickaelian, A. M. 2007, Ap 50, 127

Lasker, B. M., Lattanzi, M. G., McLean, B. J., et al. 2008, AJ, 136, 735L

Markarian, B. E. 1967, Ap 3, 24

Markarian, B. E.; Lipovetsky, V. A.; Stepanian, J. A.; et al. 1989, Comm. SAO 62, 5

Massaro, E.; Maselli, A.; Leto, C.; et al. 2015, Ap\&SS 357, 75

Massaro, E.; Mickaelian, A. M.; Nesci, R.;Weedman, D. (Eds.) 2008, Digitized First Byurakan Survey, ARACNE Editrice, Rome, 78 p.

McMahon, R.G., Irwin, M.J., Maddox, S.J. 2000, The APM-North Catalogue, Inst. of Astron., Cambridge, UK

Mickaelian, A. M. 1995, Ap 38, 349

Mickaelian, A. M. 1997, Ap 40, 1

Mickaelian, A. M. 2000, A\&ATr 18, 557

Mickaelian, A. M. 2001a, Proc. Conf.: The New Era of Wide-Field Astronomy. ASP Conf. Series 232, 278

Mickaelian, A. M. 2001b, Proc. IAU Symp. 204: The Extragalactic Infrared Background and its Cosmological Implications. ASP Conf. Series 204, 69

Mickaelian, A. M. 2002, Proc. IAU Col. 184: AGN Surveys. ASP Conf. Series 284, 101 
Mickaelian, A. M. 2003a, A\&ATr 22, 753

Mickaelian, A. M. 2003b, Proc. JENAM-2002, Kluwer Academic Publishers 285, 76

Mickaelian, A. M. 2004a, A\&A 426, 367

Mickaelian, A. M. 2004b, Ap 47, 361

Mickaelian, A. M. 2004c, Baltic Astronomy 13, 655

Mickaelian, A. M. 2007a, Highlights of Astronomy 14, 594

Mickaelian, A. M. 2007b, Proc. IAU Symp. 235: Galaxy Evolution across the Hubble Time. Cambridge Univ. Press 235, 225

Mickaelian, A. M. 2008, AJ 136, 946; VizieR On-line Data Catalog III/258

Mickaelian, A. M. 2014, Proc. IAU Symp. 304: Multiwavelength AGN Surveys and Studies, Cambridge Univ. Press 304, 1

Mickaelian, A. M. 2015, IrJAA 2, 1

Mickaelian, A. M. 2016, Astronomical Surveys and Big Data, Eds. A. M. Mickaelian, A. Lawrence, T. Yu. Magakian. ASP Conf. Series, Vol. 505, p. 3

Mickaelian, A. M. 2016, Astronomical Surveys and Big Data, Eds. A. M. Mickaelian, A. Lawrence, T. Yu. Magakian. ASP Conf. Series, Vol. 505, p. 117

Mickaelian, A. M. 2016, Astronomy Reports, Vol. 60, p. 857

Mickaelian, A. M. 2016, Baltic Astronomy 25, 75

Mickaelian, A. M.; Abrahamian, H. V.; Guibert, J.; Chesnel, R. 2002, Ap 45, 73

Mickaelian, A. M.; Abrahamyan, H. V.; Gyulzadyan, M. V.; Mikayelyan, G. A.; Paronyan, G. M. 2017, Proc. IAU Symposium \#325: Astroinformatics, Cambridge Univ. Press., Vol. 325, p. 32

Mickaelian, A. M.; Abrahamyan, H. V.; Gyulzadyan, M. V.; Paronyan, G. M.; Mikayelyan, G. A. 2018, MNRAS, 2018, in press.

Mickaelian, A. M.; Abrahamyan, H. V.; Gyulzadyan, M. V.; Paronyan, G. M.; Mikayelyan, G. A. 2018, Astrophysics and Space Science (ApSS), 2018, in press.

Mickaelian, A. M.; Abrahamyan, H. V.; Harutyunyan, G. S. 2015, Proc. ByurakanAbastumani Colloquium: Instability and Evolution of Stars. Yerevan, NAS RA "Gitutyun" Publ. House, p. 109

Mickaelian, A. M.; Abrahamyan, H. V.; Harutyunyan, G. S.; Paronyan, G. M. 2014, Proc. IAU Symp. 304: Multiwavelength AGN Surveys and Studies. Cambridge Univ. Press 304, 41

Mickaelian, A. M.; Abrahamyan, H. V.; Paronyan, G. M. 2015, Proc. IAU Symp. 319, Cambridge Univ. Press, p. 34

Mickaelian, A. M.; Abrahamyan, H. V.; Paronyan, G. M.; Harutyunyan, G. S. 2012, Proc. IAU Symp. 284: The Spectral Energy Distribution of Galaxies. Cambridge Univ. Press 284,237 
Mickaelian, A. M.; Abrahamyan, H. V.; Paronyan, G. M.; Harutyunyan, G. S. 2013, AstronomischeNachrichten 334, 887

Mickaelian, A. M.; Balayan, S. K.; Hakopian, S. A. 2001, Astron. Astrophys. Trans. 20, 315

Mickaelian, A. M.; Balayan, S. K.; Hakopian, S. A. 2002, Proc. IAU Col. 184: AGN Surveys. ASP Conf. Series 284, 217

Mickaelian, A. M.; Gonçalves, A. C.; Véron-Cetty, M. P.; Véron, P. 1999, Ap 42, 1

Mickaelian, A. M.; Gonçalves, A. C.; Véron-Cetty, M. P.; Véron, P. 2001, Ap 44, 14

Mickaelian, A. M.; Gyulzadyan, M. V.; Abrahamyan, H. V.; Paronyan, G. M.; Mikayelyan,

G. A. 2017, Proc. International Conference "Non-Stable Universe: Energetic Resources, Activity Phenomena and Evolutionary Processes", Eds. A. M. Mickaelian, H. A. Harutyunian, E. H. Nikoghosyan, ASP Conf. Series, Vol. 511, p. 149

Mickaelian, A. M.; Hakopian, S. A.; Balayan, S. K. 1999, Proc. IAU Symp. 194: Activity in Galaxies and Related Phenomena. ASP Conf. Series 194, 156

Mickaelian, A. M.; Hakopian, S. A.; Balayan, S. K. 2002, Proc. IAU Col. 184: AGN Surveys. ASP Conf. Series 284, 220

Mickaelian, A. M.; Hakopian, S. A.; Balayan, S. K.; Burenkov, A. N. 1998, Astron. Letters 24,635

Mickaelian, A. M.; Hakopian, S. A.; Balayan, S. K.; Dodonov, S. N.; Afanasiev, V. L.; Burenkov, A. N.; Moiseev, A. V. 2002, Bul. Spec. Astrophys. Obs. 53, 144

Mickaelian, A. M.; Harutyunyan, G. S. 2013a, Proc. IAU Symp. 295: The Intriguing Life of Massive Galaxies 295, 182

Mickaelian, A. M.; Harutyunyan, G. S. 2013b, Proc. IAU Symp. 292, 159

Mickaelian, A. M.; Harutyunyan, G. S.; Sarkissian, A. 2017, Astron. Letters, in press.

Mickaelian, A. M.; Hovhannisyan, L. R.; Engels, D.; Hagen, H.; Voges, W. 2006, A\&A 449,425

Mickaelian, A. M.; Hovhannisyan, L. R.; Sargsyan, L. A. 2003, Ap 46, 177

Mickaelian, A. M.; Mikayelyan, G. A.; Sinamyan, P. K. 2011, MNRAS 415, 1061

Mickaelian, A. M.; Nesci, R.; Rossi, C.; et al. 2007, A\&A 464, 1177

Mickaelian, A. M.; Paronyan, G. M. 2014, Proc. Byurakan-Abastumani Colloquium: Instability and Evolution of Stars. Yerevan, NAS RA "Gitutyun" Publ. House, p. 77

Mickaelian, A. M.; Paronyan, G. M.; Abrahamyan, H. V. 2016, Astron. Focus 2

Mickaelian, A. M.; Paronyan, G. M.; Abrahamyan, H. V.; Gyulzadyan, M. V.; Mikayelyan, G. A. 2016, Proc. Armenian-Iranian Astronomical Workshop (AIAW), Eds.: A. M. Mickaelian, H. G. Khosroshahi, H. A. Harutyunian. Yerevan, NAS RA "Gitutyun" Publ. House, p. 170

Mickaelian, A. M.; Paronyan, G. M.; Harutyunyan, G. S.; Abrahamyan, H. V.; Gyulzadyan, M. V. 2016, A\&ApTr 29, 333 
Mickaelian, A. M.; Paronyan, G. M.; Harutyunyan, G. S.; Abrahamyan, H. V.; Gyulzadyan, M. V. 2016, Astronomical and Astrophysical Transactions29, p. 333

Mickaelian, A. M.; Sargsyan, L. A. 2004, Ap 47, 213

Mickaelian, A. M.; Sargsyan, L. A. 2010, Ap 53, 483

Mickaelian, A. M.; Sargsyan, L. A., Mikayelyan G.A. 2010, Proc. IAU Symp. 267: Coevolution of Central Black Holes and Galaxies. Cambridge Univ. Press 267, 124

Mickaelian, A. M.; Sargsyan, L. A.; Astsatryan, H. V.; Cirimele, G.; Nesci, R. 2009, Data Science J. 8, 152

Mickaelian, A. M.; Sargsyan, L. A.; Gigoyan, K. S.; et al. 2009, Romanian Astron. J. 18S, 249

Mickaelian, A. M.; Sinamyan, P. K. 2010, MNRAS 407, 681

Mickaelian, A. M.; Véron-Cetty, M. P.; Véron, P. 2001, Proc. IAU Symp. 205: Galaxies and their Constituents at the Highest Angular Resolutions. ASP Conf. Series 205, 232

Mickaelian, A.; Abrahamyan, H.; Paronyan, G. 2016, Galaxies at High Redshift and Their Evolution Over Cosmic Time, IAU Symp. Vol. 319, p. 34

Mickaelian, A.; Lawrence, A.; Magakian, T. (Eds.) 2016, Astronomical Surveys and Big Data, ASP Conf. Series, Vol. 505, 290 p.

Mickaelian, A.; Paronyan, G.; Abrahamyan, H. 2016, Astronomy in Focus. Proc. IAU XXIX General Assembly, Vol. 29B, p. 91

Monet, D. G.; Levine, S. E.; Canzian, B.; et al. 2003, AJ 125, 984

Monet, D.; Bird, A.; Canzian, B.; et al. 1998, USNO Flagstaff Station and Universities Space Research Association (USRA), Vizier online catalogue I/252

Moshir, M., Kopan, G., Conrow, T., et al. 1990, IRAS FSC, Version 2.0, NASA

Pâris, I.; Petitjean, P.; Aubourg, É.; et al. 2014, A\&A 563, 54

Paronyan G. M., Abrahamyan H. V., Harutyunyan G. S., Mickaelian, A. M. 2014, Proc. IAU Symp. 304: Multiwavelength AGN Surveys and Studies. Cambridge Univ. Press 304, 164

Paronyan, G. M.; Harutyunyan G. S.; Mickaelian, A. M. 2014, Proc. IAU Symp. 304: Multiwavelength AGN Surveys and Studies. Cambridge Univ. Press 304, 166

Paronyan, G. M.; Mickaelian, A. M. 2012, Proc. Conf.: 50 years of Cosmic Era: Real and Virtual Studies of the Sky. Yerevan, NAS RA, p. 202

Paronyan, G. M.; Mickaelian, A. M. 2015, Commun. Pulkovo MAO 222, 77

Paronyan, G. M.; Mickaelian, A. M. 2018, Ap\&SS, in press.

Paronyan, G. M.; Mickaelian, A. M.; Abrahamyan H. V. 2014, Proc. IAU Symp. 304: Multiwavelength AGN Surveys and Studies. Cambridge Univ. Press 304, 161

Paronyan, G. M.; Mickaelian, A. M.; Abrahamyan, H. V. 2016, Astronomical Surveys and Big Data, Eds. A. M. Mickaelian, A. Lawrence, T. Yu. Magakian. ASP Conf. Series, Vol. 505, p. 189 
Sargsyan, L. A.; Mickaelian, A. M. 2006, Ap 49, 19

Sargsyan, L. A.; Mickaelian, A. M.; Weedman, D.; Houck, J. 2008, ApJ 683, 114

Sargsyan, L. A.; Mickaelian, A. M.; Weedman, D.; Houck, J. 2010, Proc. Conf.: Evolution of Cosmic Objects through their Physical Activity. Yerevan, NAS RA "Gitutyun" Publ. House, p. 231

Sargsyan, L. A.; Weedman, D.; Lebouteiller, V.; Houck, J.; Barry, D.; Hovhannisyan A.; Mickaelian, A. M. 2011, ApJ 730, 19

Schmidt, M.; Green, R. F. 1983, ApJ 269, 352

Skrutskie, M. F.; Cutri, R. M.; Stiening, R.; et al. 2006, AJ 131, 1163

Véron, P.; Lindblad, P. O.; Zuiderwijk, E. J.; Véron, M. P.; Adam, G. 1980, A\&A 87, 245

Véron, P.; Mickaelian, A. M.; Gonçalves, A. C.; Véron-Cetty, M. P. 1999, Proc. IAU Symp. 194: Activity in Galaxies and Related Phenomena. ASP Conf. Series 194, 140

Véron-Cetty, M. P.; Balayan, S. K.; Mickaelian, A. M.; Mujica, R.; Chavushyan, V. H.; Hakopian, S. A.; Engels, D.; Véron, P.; Zickgraf, F.-J.; Voges, W.; Xu, D.-W. 2004, A\&A 414,487

Véron-Cetty, M.-P.; Véron, P. 2010, A\&A 518, 10

Voges, W., Aschenbach, B., Boller, T., et al. 1999, A\&A, 349, 389

Voges, W., Aschenbach, B., Boller, T., et al. 2000, MPE Garching

Yamamura, I.; Makiuti, S.; Ikeda, N.; et al. 2010, AKARI/FIS All-Sky Survey Point Source Catalogues 